\title{
Hitting the CRISPR target
}

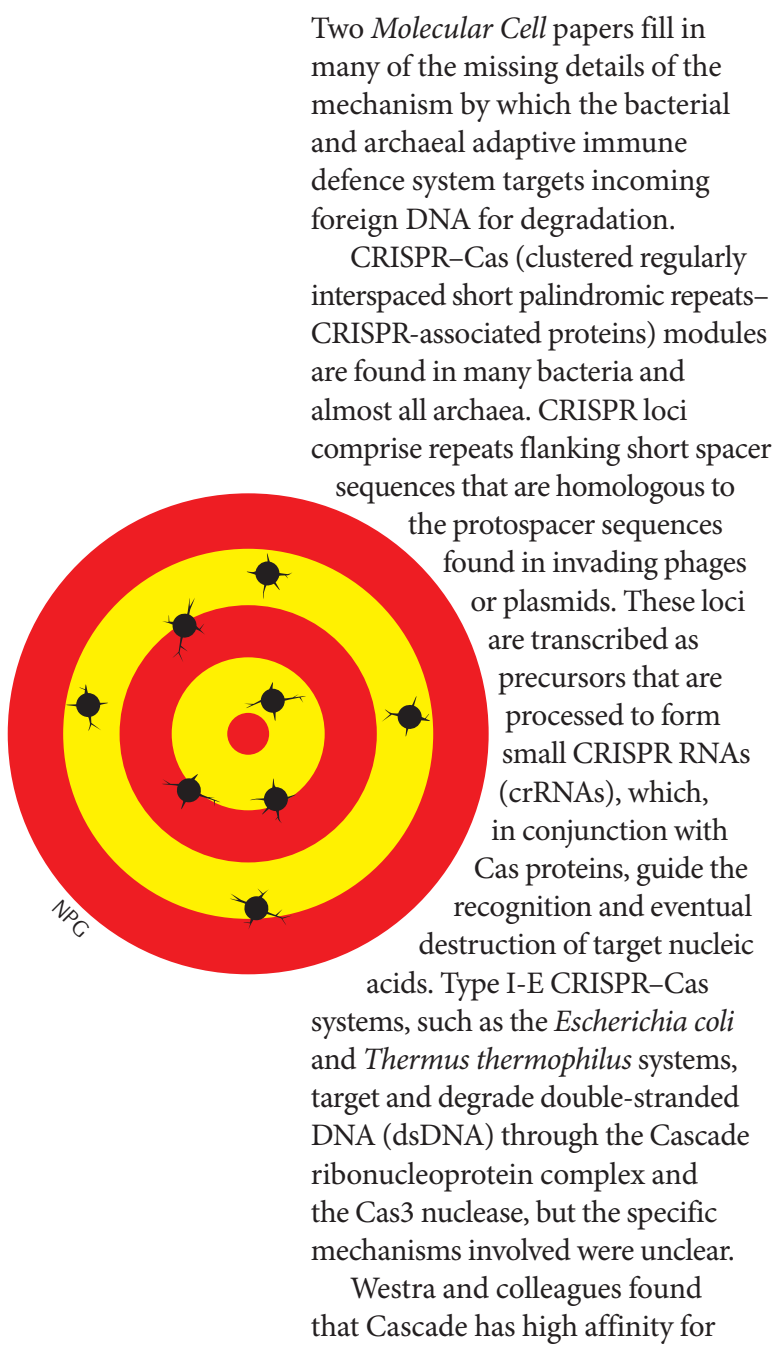

negatively supercoiled dsDNA substrates. Previous work had suggested that a motif in the target DNA known as the protospacer-adjacent motif (PAM) was important for target binding. Using gel shift assays to assess Cascade binding to various constructs, Westra et al. found that PAM is indeed crucial for Cascade binding to its target duplex and that PAM recognition takes place on the strand complementary to the crRNA. What about target DNA degradation? The authors used bimolecular fluorescence complementation to monitor the interactions between Cascade, Cas 3 and target DNA in vivo and found that Cascade interacts with Cas 3 following protospacer recognition and that Cas 3 is recruited by the Cse1 subunit (also known as CasA). The target DNA is then cleaved by the HD nuclease domain of Cas3 and undergoes ATP-dependent unwinding and degradation.

Sashital et al. focused on the role of Cse1. Previous cryo-electron microscopy (cryo-EM) analyses had suggested that Cse1 was involved in both specific and nonspecific DNA binding. The authors first undertook a biochemical analysis, which showed that Cse1 is required for Cascade to bind its target dsDNA. The authors went on to solve the structure of
T. thermophilus Cse1 to $2.3 \AA$ resolution. The structure was docked into a cryo-EM reconstruction of the $E$. coli Cascade complex, which revealed that a conformational shift on target binding positions a flexible loop (L1) in the amino-terminal domain so that it is adjacent to the crRNA, and that L1 could also contact the flanking PAM sequence. The importance of L1 was probed further by in vitro mutational analysis, which identified a conserved 3 amino acid motif that was shown to be crucial for dsDNA binding. In agreement with Westra et al., the authors found that PAM recognition by Cse1 occurred on the strand complementary to the crRNA.

Both groups present a model in which the Cascade nucleoprotein complex first binds nonspecifically to the target DNA and scans for a PAM sequence before binding specifically to the target protospacer and initiating degradation of the invading DNA.

Sheilagh Molloy

ORIGINAL RESEARCH PAPERS Westra, E. R. et al. CRISPR immunity relies on the consecutive binding and degradation of negatively supercoiled invader DNA by Cascade and Cas3. Mol. Cell 19 Apr 2012 (doi:10.1016/j.molcel.2012.03.018) | Sashital, D. G., Wiedenheft, B. \& Doudna, J. A. Mechanism of foreign DNA selection in a bacterial adaptive immune system. Mol. Cell 19 Apr 2012 (doi:10.1016/j.molcel.2012.03.020) 